\title{
Review
}

\section{Maternal-fetal fluid balance and aquaporins: from molecule to physiology}

\author{
Xiao-yan SHA ${ }^{1}$, Zheng-fang XIONG ${ }^{1}$, Hui-shu LIU ${ }^{1, *}$, Xiao-dan $\mathrm{DI}^{1}$, Tong-hui MA ${ }^{2}$ \\ ${ }^{1}$ Department of Obstetrics, Guangzhou Women and Children's Medical Centre, Guangzhou Medical College, Guangzhou 510623, \\ China; ${ }^{2}$ Central Research Laboratory, Jilin University Bethune Second Hospital, Changchun 130024, China
}

\begin{abstract}
Maternal-fetal fluid balance is critical during pregnancy, and amniotic fluid is essential for fetal growth and development. The placenta plays a key role in a successful pregnancy as the interface between the mother and her fetus. Aquaporins (AQPs) form specific water channels that allow the rapid transcellular movement of water in response to osmotic/hydrostatic pressure gradients. AQPs expression in the placenta and fetal membranes may play important roles in the maternal-fetal fluid balance.
\end{abstract}

Keywords: aquaporin; placenta; fetal membranes; maternal-fetal fluid balance

Acta Pharmacologica Sinica (2011) 32: 716-720; doi: 10.1038/aps.2011. 59; published online 23 May 2011

\section{Introduction}

Water is the major component of cells and tissues. Although adults contain $55 \%-65 \%$ water, the fetal body consists of about $70 \%-90 \%$ water, with a lower percentage close to term ${ }^{[1,2]}$. Water across the plasma membrane of cells is a fundamental activity of life, and water homeostasis during fetal development is of crucial physiological importance. Fluid balance in the fetus is dependent on its mother. For example, when the mother is dehydrated, the fetal plasma osmolality increases in parallel with the maternal plasma osmolality when the placental function is normal ${ }^{[3]}$. Maternal-fetal fluid exchange at the placenta and fetal membranes and through one pathway of exchange between the fetus and amniotic fluid can occur across the skin before full keratinization. Abortion, premature birth, amniotic fluid volume abnormality, malformation and fetal growth restrictions may result when the homeostasis of the maternal-fetal fluid exchange is disrupted. Thus, maternal-fetal fluid balance is critical during pregnancy.

The molecular mechanisms of maternal-fetal fluid balance are not known. According to researchers ${ }^{[4-6]}$, several mechanisms, including aquaporins, hormones, blood pressure differences, vascular endothelial growth factor (VEGF) and behavioral regulation, play important roles in maternal-fetal fluid balance. Hormonal mechanisms include the renin-angiotensin system, aldosterone and vasopressin regulation, which

\footnotetext{
* To whom correspondence should be addressed.

E-mail huishuliu@hotmail.com

Received 2011-02-15 Accepted 2011-04-18
}

are involved in the alteration of fetal renal excretion, water and sodium reabsorption, and the regulation of vascular volume. In addition, elevated levels of angiotensin II in the fetus increase blood pressure and cause diuresis, which contribute to an increase in amniotic fluid volume. Blood pressure regulation also plays a role in sodium/water homeostasis. VEGF is involved in the regulation of intramembranous blood vessel proliferation, membrane transport via passive permeation and the non-passive transcytotic vesicular movement of fluid. In utero behavioral regulations, such as fetal swallowing, are early functional developments in response to dipsogens. Several aquaporins are expressed in placenta, and aquaporins play key roles in the placental mechanism.

Aquaporins (AQPs) are small (about $30 \mathrm{kDa}$ ) membrane proteins that are named for their ability to increase the water permeability of the lipid bilayer of plasma membranes. There are 13 known mammalian AQPs, and certain AQPs increase the permeability to small molecules, such as glycerol (AQP3, $7,9)$, urea $(\mathrm{AQP} 3,7,8,9)$ and ammonia $(\mathrm{AQP} 8)^{[7]}$. AQPs are distributed in different cells in various organs and play critical roles in water and other small uncharged molecules transport across cell membranes ${ }^{[8-14]}$. AQPs facilitate transepithelial fluid transport and are involved in a variety of physiological and cellular functions, such as peritoneal dialysis ${ }^{[15]}$, pleural fluid transport ${ }^{[16]}$, intraocular pressure and aqueous fluid production $^{[17]}$, corneal endothelium fluid transport ${ }^{[18]}$ and amniotic fluid volume ${ }^{[19]}$.

This review discusses the role of AQPs in maternal-fetal fluid balance. The location, expression and regulation of 
AQPs in the female reproductive system, placenta and fetal membranes, abnormal amniotic fluid volume combined with direct evidence from AQP knockout mice (AQP-KO) support that AQPs play important roles in maternal-fetal fluid balance.

\section{AQP expression in the female reproductive system}

All living things reproduce. The reproductive system is essential for species survival, but it is not essential to keep an individual alive. A normal reproductive system is the basis of pregnancy and is fundamental for the maternal-fetal fluid balance.

Several subtypes of AQPs have been documented in the reproductive system of both male and female humans, rats and mice ${ }^{[19-28]}$. In the female reproductive system, AQPs are strongly expressed in the ovary, oviduct, uterus, placenta, amnion and chorion during pregnancy ${ }^{[19-22,25-28]}$. At least nine AQP isoforms (AQP1-9) are expressed in these organs (Figure $1)$.

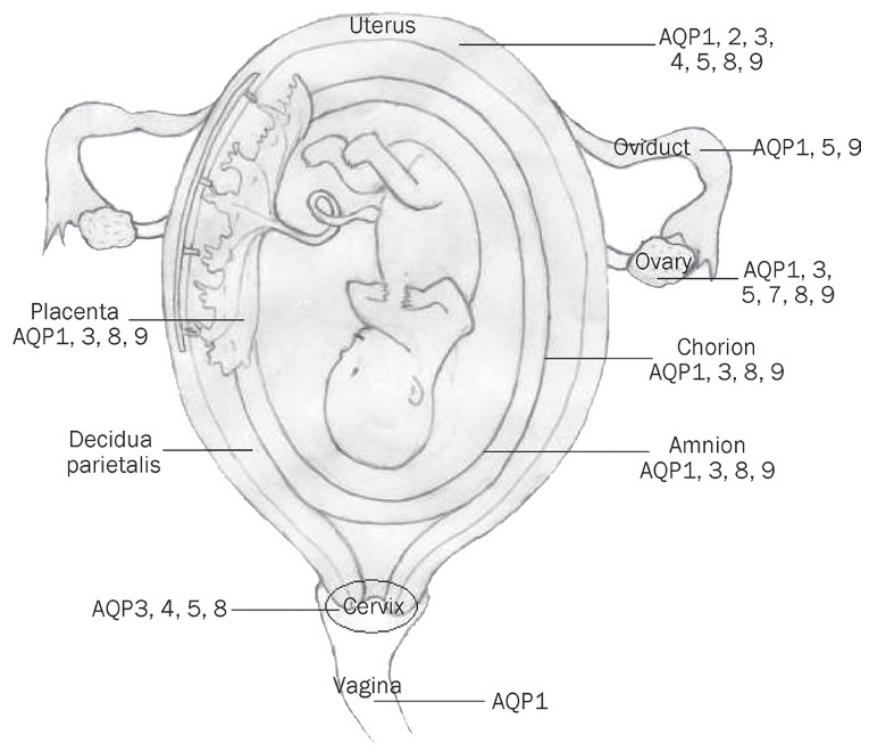

Figure 1. AQPs expression in pregnant female mammalian reproductive system.

The location and expression of AQPs in the female reproductive system suggest that AQPs play important roles in the production of ovum, the secretion of hormones, the regulation of the success of fertilization and early embryonic development ${ }^{[29-31]}$. Moreover, these functions of AQPs indicate an involvement in maternal-fetal fluid homeostasis.

\section{Placenta (pregnant temporary organ)}

The placenta is a remarkable organ between the mother and her fetus and plays a key role in ensuring a successful pregnancy. During its relatively short life span, the placenta undergoes rapid growth, differentiation and maturation. Nearly all materials that are exchanged between mother and fetus occur at the placenta, and all transport across the placenta must occur across the syncytial covering of the villous tree, the syncytiotrophoblast, the villous matrix and the fetal endothelium, each of which may impose its own restrictions and selectivity.

\section{Placental transfer}

The tissue that separates maternal and fetal blood in the placenta is called the placental barrier. The mature human placenta (hemochorial type) is a discoid organ with an elaborately branched fetal villous tree that is bathed directly by maternal blood. Primates, rodents and lagomorphs have placentas of the hemochorial type.

Paracellular and transcellular are the two pathways of transference across the placenta. The paracellular pathway across the placenta is based on the observation that the placenta is permeable to inert hydrophilic solutes that do not enter cells (eg, inulin), and its existence in the hemochorial placenta is generally accepted ${ }^{[32]}$. However, the placental barrier includes a layer of continuous trophoblast syncytium. Therefore, the transcellular route is very important for placental transfer. The existence of transtrophoblastic channels has been demonstrated $^{[33,34]}$

In the transcellular route, molecules pass through the plasma membranes of the cells that constitute the barrier. A transcellular pathway is available for substances such as lipidsoluble molecules, very small hydrophilic molecules and membrane carriers and channels.

Water is transferred across the hemochorial placenta through both the paracellular and transcellular routes, and its transfer may be facilitated by integral membrane water channel proteins (ie, AQPs).

\section{Amniotic fluid (AF) circulation}

Because amniotic fluid volume regulation is a key part of the maternal-fetal fluid balance, it has been the focus of research. The amniotic fluid serves as a significant extracorporeal water store for fetal development, including normal anatomic and fetal lung development, and as protection from fetal trauma. A normal amniotic fluid volume is critical for normal fetal growth and symmetrical development. Insufficient (oligohydramnios) or excessive (polyhydramnios) amniotic fluid volume is associated with impaired fetal outcome, including fetal structural or functional abnormalities.

Amniotic fluid volume is dependent on gestational age, and a high regulatory ability maintains the amniotic fluid volume within a fixed range ${ }^{[35]}$. Amniotic fluid pathways include the production of fetal urine, fetal swallowing, fetal lung secretion and intramembranous and transmembranous pathways ${ }^{[36,37]}$.

A variety of factors, such as postmaturity syndrome, maternal disease, maternal medications, altitude, fetal malformations and abnormal fetal weight, may affect amniotic fluid volume $^{[36]}$. Although the regulation of amniotic fluid circulation is poorly understood, the flow of water across biological membranes and the function of membrane water channels is involved. 


\section{AQP and maternal-fetal fluid balance}

\section{AQP expression in placenta and fetal membranes}

Placentation varies in rodents, sheep and humans. Consistent with previous research, our studies ${ }^{[38-41]}$ have demonstrated that AQP1, 3, 8, and 9 are the major AQPs in the placenta and fetal membranes ${ }^{[19]}$. This localization indicates a possible functional role in fluid homeostasis. The location of AQPs in placenta and fetal membranes are summarized in Table 1.

Table 1. AQPs location in placenta and fetal membranes of human, mouse and ovine.

\begin{tabular}{|c|c|c|c|}
\hline AQP & Species & Location & References \\
\hline \multirow[t]{7}{*}{ AQP1 } & Human & vascular endothelial cell, & [38] \\
\hline & & syncytiotrophoblasts, & [44] \\
\hline & & $\begin{array}{l}\text { epithelial cells of the amnion, } \\
\text { cytotrophoblasts of the chorion }\end{array}$ & \\
\hline & Mouse & amnion, & [19] \\
\hline & & vessel walls of placental labyrinth & \\
\hline & Ovine & vascular endothelial cells, & \\
\hline & & capillary endothelium of the cotyledons & {$[14]$} \\
\hline \multirow[t]{7}{*}{ AQP3 } & Human & placenta syncytiotrophoblasts, & [39] \\
\hline & & cytotrophoblasts, & {$[55]$} \\
\hline & & chorion cytotrophoblasts, & [56] \\
\hline & Mouse & amnion, & \\
\hline & & labyrinth trophoblast cells & [14] \\
\hline & Ovine & cytotrophoblasts of chorion and placenta, & \\
\hline & & fibroblasts of amnion and allantois & [19] \\
\hline \multirow[t]{2}{*}{ AQP8 } & Human & amnion and chorion epithelial cells, & [28] \\
\hline & & trophoblasts & [40] \\
\hline \multirow[t]{3}{*}{ AQP9 } & Human & amnion epithelial cells, & [41] \\
\hline & & $\begin{array}{l}\text { cytotrophoblasts of chorion, } \\
\text { trophoblasts }\end{array}$ & [56] \\
\hline & Ovine & epithelial cells amnion and allantois & [57] \\
\hline
\end{tabular}

AQP expression regulation in placenta and fetal membranes Ontogeny expression of AQPs in placenta and fetal membranes

Several AQP subtypes are expressed in placenta and fetal membranes, and an alteration in the expression of AQPs has been detected during pregnancy. Our previous study ${ }^{[14]}$ showed that the ontogeny of mRNA expression for AQP1, 3 and 8 in the ovine placenta at different gestational ages $(27,45$, 66,100 , and $140 \mathrm{~d}$, where term is $\sim 150 \mathrm{~d}$ ). AQP1 was the only aquaporin present in the vasculature and was significantly higher at $27 \mathrm{~d}$ of gestation compared to the other time points. This result suggested that AQP1 might be related to placental angiogenesis. AQP3 was increased significantly across the gestational period, and the increase in expression coincided with a substantial increase in urea permeability in the ovine placenta.

Beall et $a l^{[19]}$ demonstrated that advancing gestation was associated with an increase in amniotic fluid volume from gestational days e10 to e16 with a marked decrease in amniotic fluid volume from e16 to e19. Fetal membrane AQP1, placental AQP1 and AQP9 expression were negatively correlated with amniotic fluid volume, and placental AQP3 expression was positively correlated with amniotic fluid volume.

The above results indicate that AQPs play roles in placental development and placental functions. Moreover, these results imply that the ontogeny of AQP expression is related to maternal-fetal fluid balance.

\section{AQP expression in placenta with abnormal amniotic fluid volume}

Amniotic fluid is essential for the developing fetus to provide the appropriate aquatic environment for symmetrical and normal development. Abnormal amniotic fluid volume induces premature delivery, fetal growth restriction, fetal distress, meconium aspiration syndrome, malformations and fetal death $^{[42,43]}$. Compared to normal amniotic fluid volume, Mann et $a l^{[44]}$ detected a significant increase in AQP1 expression, particularly in the amnion (33-fold) of polyhydramnios. Zhu et $a l^{[45]}$ also indicated that there was a significant decrease of AQP1 and AQP3 expression in the amnion and chorion of the oligohydramnios group, but AQP3 expression in placenta was significantly increased compared to the normal amniotic fluid volume group.

Our preliminary studies ${ }^{[46-48]}$ have shown that the expression of AQP1 mRNA is significantly lower in oligohydramnios placenta and fetal membranes than in normal pregnancy at term. The expression of AQP8 mRNA is significantly lower in oligohydramnios placenta than in normal pregnancy placenta at term. The expression of AQP9 mRNA in fetal membranes is significantly higher in polyhydramnios groups than in controls. AQP1, 3, 8, and 9 may play important roles in the maintenance of amniotic fluid volume and the balance of different components in oligohydramnios and polyhydramnios patients.

The above results indicate that the change in AQP expression in human placenta is related to amniotic fluid volume regulation. Furthermore, more evidence at the molecular level demonstrates that AQPs have an important effect on amniotic fluid volume and maternal-fetal fluid balance.

\section{Direct evidence from AQP gene knockout mice}

The AQP gene knockout mice (AQP-KO) have been studied for years and have provided direct evidence of their physiological functions. Several analysis of AQP-KO mice have revealed that AQP-knockout mice induce unexpected physiological changes, including an impairment of angiogenesis and cell migration ${ }^{[49]}$, saliva secretion disability ${ }^{[50]}$, cerebrospinal fluid (CSF) dynamics ${ }^{[51]}$, peritoneal dialysis ${ }^{[52]}$, urinary concentrating ability disturbances ${ }^{[53,54]}$ and polyhydramnios ${ }^{[20]}$.

AQP1 plays an important role in angiogenesis and endothelial cell migration. In AQP1-knockout mice, aortic endothelia migration and wound healing is greatly impaired, but abnormal vessel formation is observed in vitro. The mechanism proposed is that water influx at the tip of a lamellipodium results 
in membrane protrusion in the direction of cell migration ${ }^{[49]}$. AQPs play roles in saliva secretion by transepithelial fluid transport. Osmotic equilibration is impaired in AQP5-knockout mice, which results in a reduced volume of relatively hypertonic fluid secretion ${ }^{[50]}$. AQP1 is strongly expressed at the ventricular facing surface of the choroids plexus epithelium (CPE). CSF production and intracranial pressure (ICP) are decreased in AQP1-knockout mice. Water transport by AQP1 is a substantial percentage of choroidal CSF production, and a deficiency of AQP1-mediated transcellular routes may contribute to the decrease in CSF following a reduction in $\mathrm{ICP}^{[51]}$. AQP1-knockout mice show a decreased initial and cumulative ultrafiltration (UF) without sodium sieving during peritoneal dialysis (PD). This result supports the essential three-pore theory and a similar mechanism in the descending vasa recta $^{[52]}$. AQP1 is also strongly expressed in the proximal tubule of the kidney, the descending limb of Henle epithelia and in the vasa recta endothelia. Urinary concentrating ability is severely impaired in AQP1-knockout mice. The primary renal defect in AQP1 knockout mice is the inability to generate a hypertonic medullary interstitium by countercurrent multiplication $^{[53,54]}$.

Mann et $a l^{[20]}$ have shown that AQP1 gene knockout mice have a greater amniotic fluid volume and lower amniotic fluid osmolality than wild-type and heterozygote counterparts. The result represents the movement of water through the AQP1 channel because of the impaired renal fluid absorption and concentrating ability in AQP1-knockout mice. Moreover, deficiency in the regulation of water movement across the fetal membranes and within the placental trophoblast may be another mechanism for this result. AQP1 in fetal membranes may contribute to amniotic fluid volume regulation, and it is speculated that idiopathic polyhydramnios may be associated with a deficiency in AQP1 in human fetal membranes.

Our research in AQP-knockout pregnant mice is consistent with the above results. For example, our study showed an increase in embryo numbers, heavier placental and fetal/ neonatal weight and an increase in the amount of amniotic fluid in AQP8-knockout pregnant mice ${ }^{[58]}$. The results of the pregnant phenotypes of AQP-knockout mice provide direct evidence that AQPs play important roles in pregnancy, fetal growth and maternal-fetal fluid balance. Therefore, screening for aquaporin mutations in genetic diseases associated with abnormalities in fluid balance may be required.

In summary, successful pregnancy requires high-quality ovulation, successful fertilization and normal embryonic and fetal development, in which water homeostasis plays a key role throughout pregnancy. The location, expression and regulation of AQPs in the female reproductive system, placenta and fetal membranes, coupled with direct evidence from AQP-knockout mice support the involvement of AQPs in this physiological process and maternal-fetal fluid balance.

\section{Acknowledgements}

This study was supported by the National Natural Science Foundation of China (30471828 and 30973206) and the Scien- tific Research Foundation for the Returned Overseas Chinese Scholars, State Education Ministry [2005]383.

\section{References}

1 Greizerstein HB. Placental and fetal composition during the last trimester of gestation in the rat. Biol Reprod 1982; 26: 847-53.

2 Engle WA, Lemons JA. Composition of the fetal and maternal guinea pig throughout gestation. Pediatr Res 1986; 20: 1156-60.

3 Wintour EM. Water and electrolyte metabolism in the fetal-placental unit. In: Cowett RM, editor. Principles of perinatal and neonatal metabolism. Second edition. New York: Springer; 1998. p 511-34.

4 Modena AB, Fieni S. Amniotic fluid dynamics. Acta Biomed 2004; 75 : 11-3.

5 Moritz K, Koukoulas I, Albiston A, Wintour EM. Angiotensin II infusion to the midgestation ovine fetus: effects on the fetal kidney. Am J Physiol Regul Integr Comp Physiol 2000; 279: R1290-7.

6 Daneshmand SS, Cheung CY, Brace RA. Regulation of amniotic fluid volume by intramembranous absorption in sheep: role of passive permeability and vascular endothelial growth factor. Am J Obstet Gynecol 2003; 188: 786-93.

7 Saparov SM, Liu K, Agre P, Pohi P. Fast and selective ammonia transport by aquaporin-8. J Biol Chem 2007; 282: 5296-301.

8 Calamita G, Mazzone A, Bizzoca A, Svelto M. Possible involvement of aquaporin-7 and -8 in rat testis development and spermatogenesis. Biochem Biophys Res Commun 2001; 288: 619-25.

9 Calamita G, Mazzone A, Cho YS, Valenti G, Svelto M. Expression and localization of the aquaporin-8 water channel in rat testis. Biol Reprod 2001; 64: 1660-6.

10 Elkjaer ML, Nejsum LN, Gresz V, Kwon TH, Jensen UB, Frokiaer J, et al. Immunolocalization of aquaporin-8 in rat kidney, gastrointestinal tract, testis, and airways. Am J Physiol Renal Physiol 2001; 281: F1047-57.

11 Hoque AT, Yamano S, Liu X, Swaim WD, Goldsmith CM, Delporte C, et al. Expression of the aquaporin 8 water channel in a rat salivary epithelial cell. J Cell Physiol 2002; 191: 336-41.

12 Hurley PT, Ferguson CJ, Kwon TH, Andersen ML, Norman AG, Steward $\mathrm{MC}$, et al. Expression and immunolocalization of aquaporin water channels in rat exocrine pancreas. Am J Physiol Gastrointest Liver Physiol 2001; 280: G701-9.

13 Tani T, Koyama Y, Nihei K, Hatakeyama S, Ohshiro K, Yoshida Y, et al. Immunolocalization of aquaporin-8 in rat digestive organs and testis. Arch Histol Cytol 2001; 64: 159-68.

14 Liu H, Koukoulas I, Ross MC, Wang S, Wintour EM. Quantitative comparison of placental expression of three aquaporin genes. Placenta 2004; 25: 475-8.

$15 \mathrm{Ni}$ J, Verbavatz JM, Rippe A, Boisdé I, Moulin P, Rippe B, et al. Aquaporin-1 plays an essential role in water permeability and ultrafiltration during peritoneal dialysis. Kidney Int 2006; 69: 1518-25.

16 Song Y, Yang B, Matthay MA, Ma T, Verkman AS. Role of aquaporin water channels in pleural fluid dynamics. Am J Physiol Cell Physiol 2000; 279: C1744-50.

17 Zhang D, Vetrivel L, Verkman AS. Aquaporin deletion in mice reduces intraocular pressure and aqueous fluid production. J Gen Physiol 2002; 119: 561-9.

18 Kuang K, Yiming M, Wen Q, Li Y, Ma L, Iserovich P, et al. Fluid transport across cultured layers of corneal endothelium from aquaporin-1 null mice. Exp Eye Res 2004; 78: 791-8.

19 Beall MH, Wang S, Yang B, Chaudhri N , Amidi F, Ross MG. Placental and membrane aquaporin water channels: correlation with amniotic fluid volume and composition. Placenta 2007; 28: 421-8.

20 Mann SE, Ricke EA, Torres EA, Taylor RN. A novel model of polyhydramnios: amniotic fluid volume is increased in aquaporin 1 knock- 
out mice. Am J Obstet Gynecol 2005; 192: 2041-4.

21 Wang S, Chen J, Au KT, Ross MG. Expression of aquaporin 8 and its up-regulation by cyclic adenosine monophosphate in human WISH cells. Am J Obstet Gynecol 2003; 188: 997-1001

22 Anderson J, Brown N, Mahendroo MS, Reese J. Utilization of different aquaporin water channels in the mouse cervix during pregnancy and parturition and in models of preterm and delayed cervical ripening. Endocrinology 2006; 147: 130-40.

23 Yeung $\mathrm{CH}$, Callies C, Rojek A, Nielsen S, Cooper TG. Aquaporin isoforms involved in physiological volume regulation of murine spermatozoa. Biol Reprod 2009; 80: 350-7.

24 Yeung $\mathrm{CH}$, Callies C, Tüttelmann F, Kliesch S, Cooper TG. Aquaporins in the human testis and spermatozoa - identification, involvement in sperm volume regulation and clinical relevance. Int J Androl 2010; 33: 629-41.

25 McConnell NA, Yunus RS, Gross SA, Bost KL, Clemens MG, Hughes FM Jr. Water permeability of an ovarian antral follicle is predominantly transcellular and mediated by aquaporins. Endocrinology 2002; 143: 2905-12.

26 Brañes MC, Morales B, Ríos M, Villalón MJ. Regulation of the immunoexpression of aquaporin 9 by ovarian hormones in the rat oviductal epithelium. Am J Physiol Cell Physiol 2005; 288: C1048-57.

27 Jablonski EM, McConnell NA, Hughes FM Jr, Huet-Hudson YM. Estrogen regulation of aquaporins in the mouse uterus: potential roles in uterine water movement. Biol Reprod 2003; 69: 1481-7.

28 Wang S, Kallichanda N, Song W, Ramirez BA, Ross MG. Expression of aquaporin-8 in human placenta and chorioamniotic membranes: evidence of molecular mechanism for intramembranous amniotic fluid resorption. Am J Obstet Gynecol 2001; 185: 1226-31.

29 Su W, Qiao Y, Yi F, Guan X, Zhang D, Zhang S, et al. Increased female fertility in aquaporin 8-deficient mice. IUBMB Life 2010; 62: 852-7.

30 Edashige K, Sakamoto M, Kasai M. Expression of mRNAs of the aquaporin family in mouse oocytes and embryos. Cryobiology 2000; 40: $171-5$.

31 Skowronski MT, Kwon TH, Nielsen S. Immunolocalization of aquaporin 1,5 , and 9 in the female pig reproductive system. J Histochem Cytochem 2009; 57: 61-7.

32 Stulc J. Placental transfer of inorganic ions and water. Physiol Rev 1997; 77: 805-36.

33 Hedley R, Bradbury MW. Transport of polar non-electrolytes across the intact and perfused guinea-pig placenta. Placenta 1980; 1: 277 85.

34 Kaufmann P, Schroder H, Leichtweiss HP. Fluid shifts across the placenta. II. Fetomaternal transfer of horseradish peroxidase in the guinea pig. Placenta 1982; 3: 339-48.

35 Brace RA, Wolf EJ. Normal amniotic fluid volume changes throughout pregnancy. Am J Obstet Gynecol 1989; 161: 382-8.

36 Sherer DM. A review of amniotic fluid dynamics and the enigma of isolated oligohydramnios. Am J Perinatol 2002; 19: 253-66.

37 Underwood MA, Gilbert WM, Sherman MP. Amniotic fluid: not just fetal urine anymore. J Perinatol 2005; 25: 341-8.

38 Liu HS, Song XF, Hao RZ. Expression of aquaporin-1 in human placenta and fetal membranes. Nan Fang Yi Ke Da Xue Xue Bao 2008; 28: 333-6.

39 Liu HS, Song XF, Yuan WC, Hao RZ. Expression and distribution of aquparin-3 in human placenta and fetal membranes. Chin J Biomed Eng 2007; 13: 210-2.

40 Liu HS, Hao RZ, Song XF, Xiong ZF. Aquparin8 expression in human placenta and fetal membrane. Chin J Clin Rehab 2009; 13: 4791-5.

41 Liu HS, Song XF, Yuan WC. Expression of aquaporin 9 in human placenta and fetal membranes. Chin J Perinat Med 2007; 10: 252-6.

42 Manzanares S, Carrillo MP, González-Perán E, Puertas A, Montoya F. Isolated oligohydramniosin term pregnancy as an indication for induction of labor. J Matern Fetal Neonatal Med 2007; 20: 221-4.

43 Daneshmand SS, Cheung CY, BraceRA. Regulation of amniotic fluid volume by intramembranous absorption in sheep: role of passive permeability and vascular endothelial growth factor. Am J Obstet Gynecol 2003; 188: 786-93.

44 Mann SE, Dvorak N, Gilbert H, Taylor RN. Steady-state levels of aquaporin 1 mRNA expression are increased in idiopathic polyhydramnios. Am J Obstet Gynecol 2006; 194: 884-7.

45 Zhu XQ, Jiang SS, Zhu XJ, Zou SW, Wang YH, Hu YC. Expression of aquaporin 1 and aquaporin 3 in fetal membranes and placenta in human term pregnancies with oligohydramnios. Placenta 2009; 30 : 670-6.

46 Hao RZ, Liu HS, Xiong ZF. Expression of aquaporin-1 in human oligohydramnios placenta and fetal membranes. Nan Fang Yi Ke Da Xue Xue Bao 2009; 29: 1130-2.

47 Xiong ZF, Liu HS, Hao RZ. Expressions of aquaporin 3, 8 and 9 mRNA in human oligohydramnios placenta. Chin J Biomed Eng 2010; 16: $155-8$.

48 Liu HS, Hao RZ, Xiong ZF. Expression of aquaporin 1, 3, 8, 9 mRNA in human amniotic membranes in polyhydramnios. Chin J Perinat Med 2007; 12: 197-200.

49 Saadoun S, Papadopoulos MC, Hara-Chikuma M, Verkman AS. Impairment of angiogenesis and cell migration by targeted aquapofin-1 gene disruption. Nature 2005; 434: 786-92.

50 Ma T, Song Y, Gillespie A, Carlson EJ, Epstein CJ, Verkman AS. Defective secretion of saliva in transgenic mice lacking aquaporin- 5 water channels. J Biol Chem 1999; 274: 20071-4.

51 Oshio K, Watanabe H, Song Y, Verkman AS, Manley GT. Reduced cerebrospinal fluid production and intracranial pressure in mice lacking choroid plexus water channel Aquaporin-1. FASEB J 2005; 19: 76-8.

52 Yang B, Folkesson HG, Yang J, Matthay MA, Ma T, Verkman AS. Reduced osmotic water permeability of the peritoneal barrier in aquaporin-1 knockout mice. Am J Physiol 1999; 276: C76-81.

53 Chou CL, Knepper MA, Hoek AN, Brown D, Yang B, Ma T, et al. Reduced water permeability and altered ultrastructure in thin descending limb of Henle in aquaporin-1 null mice. J Clin Invest 1999; 103: 491-6.

54 Zhao D, Bankir L, Qian L, Yang D, Yang B. Urea and urine concentrating ability in mice lacking AQP1 and AQP3. Am J Physiol Renal Physiol 2006; 291: F429-38.

55 Wang S, Amidi F, Beall M, Gui L, Ross MG. Aquaporin 3 expression in human fetal membranes and its up-regulation by cyclic adenosine monophosphate in amnion epithelial cell culture. J Soc Gynecol Investig 2006; 13: 181-5.

56 Damiano A, Zotta E, Goldstein J, Reisin I, Ibarra C. Water channel proteins AQP3 and AQP9 are present in syncytiotrophoblast of human term placenta. Placenta 2001; 22: 776-81.

57 Wang S, Chen J, Huang B, Ross MG. Cloning and cellular expression of aquaporin 9 in ovine fetal membranes. Am J Obstet Gynecol 2005; 193: 841-8.

58 Sha XY, Xiong ZF, Liu HS, Zheng Z, Ma TH. Pregnant phenotype in aquaporin 8-dificient mice. Acta Pharmacol Sin 2011; 32: 805-16. 